\title{
Joon Hemels Divergenz und Konvergenz von Medienkultur und religiöser Kultur \\ Internationaler Kongress über Religion und \\ Medien in Antwerpen
}

Mit einem differenzierten Bild von Entwicklungen in der Gesellschaft, in der katholischen Kirche und in den Medien zeigte Kardinal Godfried Danneels von Briissel-Mechelen auf dem zweitägigen Kongress „Believin' the Media. Analysis Faith and Media: Representation and Communication Strategy" am 26. und 27. April 2007 in Antwerpen die ganze Spannweite dieser Thematik auf.

Die Marginalisierung der kirchlichen Nachrichten in der Zeitung und im Fernsehen, das übertriebene Interesse an Randnachrichten (mit einem manchmal bizarren Charakter) über kirchliche Würdenträger, der Bedeutungsverlust einer Spezialisierung auf kirchlich-religiösen Joumalismus, die anti-hierarchische Haltung unserer individualistisch geprägten Kultur, der Hang zur Personalisierung von Nachrichten, der Siegeszug der Bildkultur, die Ausdünnung der kirchlichen Zeitschriftenpresse - auf all diese und andere Themen richtete der Kardinal bei seinem Vortrag das Augenmerk. Für ihn sind diese gesellschaftlichen Entwicklungen jedoch kein Grund zu Pessimismus. Ganz im Gegenteil: Er forderte, nicht über vollendete Tatsachen und Unumkehrbares zu jammern, sondern damit leben $\mathrm{zu}$ lernen und das Positive darin $\mathrm{zu}$ sehen.

Kardinal Danneels scheint sich keine Illusionen zu machen, wie die Kirche in der heutigen Zeit wahrgenommen wird, nämlich als konservatives Bollwerk. Er nannte dazu die Stichwörter hierarchisch und undemokratisch. Er sagte, dass beide Mängel der Kirche in den Medien hochgespielt und als größeres Übel betrachtet würden, als sie es in Wirklichkeit seien. Mit großer Überzeugungskraft nannte er Beispiele dafür, wie in der Kirche Toleranz nicht nur gepredigt, sondern auch praktiziert werde. Er beschrieb die ideale Kirche als Haus mit vielen Zimmern, mit Bewohnern, die sich in Geduld üben, nach Toleranz suchen und den Beweis subtiler Demokratie liefern.

Dass kirchliche Autoritäten das Buch eines Theologen erst nach siebzehn Jahren verurteilten, betrachtete Danneels zunächst als Zeichen von Geduld mit dem Autor, fügte aber hinzu, dass man auf eine Verurteilung nach so langer Zeit besser hätte verzichten können. Der Umgang der Kirche mit Sexualität, aber auch mit Abtreibung und Sterbehilfe bekomme nach Ansicht des Kardinals mehr Aufmerksamkeit als die soziale Lehre. Da die Menschen besonders empfänglich seien für kirchliche Stel- 
lungnahmen zu Geburtenregelung, Sex und Euthanasie, müssten sich Bischöfe seines Erachtens positiv dazu äußern und darauf verzichten, allerlei Verbote auszusprechen. Das wäre möglich, wenn mehr Augenmerk auf die Werte gerichtet würde, die mit einem verantwortungsbewussten Umgang mit Sexualität, Leben und Sterben verbunden sind.

Für die Zukunft wünschte sich Danneels eine Kirche mit und von Menschen, die mit den Regeln der Medienlogik vertraut sind. Sie sollte die Bedeutung und Wirkung von (öffentlicher und interpersonaler) Kommunikation und aller ihr zur Verfügung stehenden Mittel einschätzen können und es wagen, sie kreativ zu nutzen. Die Kirche sollte nicht außerhalb der Medienkultur stehen, sondern sich den Herausforderungen der Massenmedien stellen müssen. Die Kirche muss sich diesen Medien nicht nur von ethischen Standpunkten her nähern, sondern sie als geeignetes Podium betrachten, um sich in der Öffentlichkeit darzustellen, an der öfentlichen Debatte teilzunehmen und sich auf das Bedürfnis nach Sinnstiftung einzustellen. „Die Wahrheit fragt nicht nach einem Schutzbrief", so der Kardinal in seinem Plädoyer für eine Kirche, die nicht vor den Massenmedien zurückschreckt, die es wagt, die Plausibilität ihrer Botschaft im öffentlichen Rahmen mit Selbstvertrauen und selbstbewusst zur Diskussion zu stellen und die sich nicht in eine selbst gewählte Isolation und eigene Nischenmedien zurïckzieht.

\section{Veränderungen in Kirche und Medienwelt}

Die Initiative zu dem Kongress, der im Kongresszentrum „Elzenfeld“ stattfand, ging vom Universitätszentrum Sint-Ignatius Antwerpen („Universitair Centrum Sint-Ignatius Antwerpen“, UCSIA) aus, einer NonProfit-Organisation mit einer Verwaltung, in der sowohl Mitglieder der Jesuiten-Sozietät als auch Vertreter der Universität Antwerpen sowie gesellschaftliche Organisationen vertreten sind.

In Plenar- und Parallelworkshops wurde das Thema der Konferenz aus verschiedenen Blickwinkeln betrachtet. Die meisten Redner beschrieben die große Distanz oder sogar Kluft, die zwischen den Kirchen und den Medien entstanden ist. Als Begleiterscheinung dieser Divergenz statt Konvergenz konstatierte Hans Geybels, Hochschullehrer für Kirche und Medien an der katholischen Universität Löwen, in seinem Eröffnungsvortrag, dass sich das Bild der katholischen Kirche als Institution in der Gesellschaft verschlechtert habe. Schuld daran sei die Art und Weise, in der die Kirche sowie kirchliche Amtsträger in den Nachrichten vorkommen. Die Entwicklung einer auf die Kirche abgestimmten Kommunikationsstrategie werde durch fehlende Offenheit und Transparenz er- 
schwert. Außerdem, so merkte er an, sei das Selbstbild innerhalb der Kirche verwirrt oder diffus. Es mangele an einem Konsens über das gewünschte Image. Während der beiden Kongresstage lag der Schwerpunkt vor allem auf der Darstellung von Kirche und Religion in den Medien und nicht auf dem im Untertitel genannten Thema „Strategische Kommunikation". Welche Kommunikationsstrategie für die gesamte Kirche sowie für jede einzelne Kirche angemessen ist, könnte Thema einer künftigen Konferenz des UCSIA sein. Dazu sollten außer Kommunikations- und Religionswissenschaftlern, Theologen, Religionssoziologen und -psychologen auch mehr Kommunikationsberater und Kirchensprecher als Gesprächspartner eingeladen werden.

Über die Wechselwirkung zwischen Veränderungen in der Medienwelt und der religiösen Entwicklung seit der Reformation sprach Stewart M. Hoover vom Zentrum für Medien, Religion und Kultur an der Universität von Colorado, USA. Die große Vielfalt der Medien, wie wir sie heute kennen, bietet nach Ansicht dieses amerikanischen Kommunikationswissenschaftlers Anbietern religiöser Inhalte mehr Chancen auf eine zielgerichtete Verbreitung ihrer Botschaft. Spezifische Zielgruppen von individuell Suchenden mit einem gemeinsamen Interesse oder Bedürfnis können angesprochen werden. Das Entstehen einer individualistischen Moral schließt nicht aus, dass sich eine kleinere Anzahl Menschen für gemeinsame Bedürfnisse engagiert. Individualisierung bekäme dann eine weniger negative Bedeutung und könnte umschrieben werden als Auf-die-Suche-gehen nach einer gemeinsamen Gruppenmoral, die in der Lage ist, Identität $\mathrm{zu}$ verleihen und Integration zu fördern, nachdem die herkömmlichen Verbände und Institutionen - von der traditionellen Familie bis zur Kirche - dermaßen geschwächt oder transformiert sind, dass sie ihre Bedeutung für viele Menschen verloren haben. Hoover sieht in der häufigen und vielseitigen Mediennutzung moderner Menschen, die nach Informationen, Erlebnissen und emotionaler Unterstützung suchen, die große Chance für Journalisten und Programmmacher, auf das zunächst latente und dann manifeste Bedürfnis nach Trost ("to comfort") statt Herausforderung ("to challenge") einzugehen. Mediennutzung inklusive Internet rücken nach Hoovers Ansicht auf den Platz, der einst von Religionen eingenommen wurde.

Der Gründer von „Communicatio Socialis“, P. Franz-Josef Eilers SVD, skizzierte die Entwicklung des Denkens der katholischen Kirche über Massenkommunikation („Communicatio Socialis“) seit dem Zweiten Vatikanischen Konzil. Für diejenigen, die weniger Bescheid wissen über die vom Vatikan verbreiteten Dokumente zu Medienfragen, bot sein Beitrag einen sehr guten Überblick. Gleichzeitig vermittelte er seine Erfahrung, dass auch - oder sogar - in der katholischen Kirche die Bereitschaft 
wächst, die Massenmedien sowie das Internet aus einer positiveren Grundhaltung zu beurteilen. In dem von Eilers präsentierten christlichen Kommunikationsmodell steht die Botschaft und nicht der Sender (Kommunikator) im Mittelpunkt: Letzterer spiegelt die Botschaft wider, die den Rezipienten übermittelt wird. Die katholische Kirche hat nach Eilers Einschätzung in den letzten Jahrzehnten einen wesentlichen Beitrag zur Medienerziehung (in Bezug auf die Rolle der Familie dabei) und zu einer Form der Medienethik, die weiter reicht als bis zum Formulieren von Verhaltenskodizes, geleistet.

In einem der Workshops beschäftigte sich Ineke de Feijter mit Äußerungen der katholischen und protestantischen Kirche über die Medien. Sie promovierte mit einer 2007 im Lit Verlag erschienenen Disser. tation „The Art of Dialogue. Religion, Communication and Global Media Culture". Darin plädiert sie dafür, die Kirche als Ort des Dialogs zu betrachten. Sie behauptet, dass die katholische Kirche in den letzten Jahrzehnten die besseren Dokumente über Kommunikation veröffentlicht hat. Der evangelischen Kirche hingegen scheint es mit weniger schriftlichen Äußerungen besser zu gelingen, sich positiv in den Medien zu präsentieren. Ihr Standpunkt bietet eine Anknüpfungsmöglichkeit für einen Folgekongress zum Thema "Strategische Kommunikation" in den verschiedenen Kirchen.

Der fruihere Direktor des Katholischen Fernseh- und Radiozentrums für flämische Programme, Emest Henau, betrachtete in seinem Beitrag das ambivalente Verhältnis von Kirche und Medien. Er skizzierte die Evolution der kirchlichen Haltung hinsichtlich der Medien als eine zögernde Entwicklung von Divergenz zu Konvergenz. Das führte letztendlich zu einer besonderen Aufmerksamkeit für den sozialen Charakter von Kommunikation und hat zum Ziel, den sozialen Zusammenhang $z u$ verstärken. Dieses Streben muss zur Soziallehre der Kirche passen, einen Gegenpol zur Individualisierungstendenz bilden und sich darauf richten, Solidarität statt individueller Bedürfnisbefriedigung zu fördern. Aus diesem Gedankengang heraus plädierte Henau für die Zuweisung von mehr Sendezeit für die Kirchen im öffentlich-rechtlichen Rundfunk. Gerade weil dieser nicht-kommerzielle Rundfunk in der vielbesungenen „civil society" von aktiven Bürgern funktioniert und eine vom Staat unabhängige Organisation mit einer lebhaften Diskussionskultur ist, sollten sich Kirchengemeinden in Radio und Fernsehen als lebendige Glaubensgemeinschaften präsentieren können.

Marc Hooghe, Politikprofessor in Löwen, präsentierte die Ergebnisse einer empirischen Untersuchung, wie Religionen in den Jahren 2003 bis 2006 in flämischen Medien zur Diskussion gestellt wurden. Bemerkenswert war, dass er dabei die heutige Journalistengeneration als offen und 
vorausstrebend charakterisierte, während er Kirchenführer als konservativ und institutionell ausgerichtet beschrieb. Er diagnostizierte einen Konflikt zwischen liberalen Werten ("liberal values") einerseits und konservativen Einstellungs- und Verhaltensmustern ("conservative value patterns") andererseits. Was die Berichterstattung in den Zeitungen betrifft, konstatierte Hooge übermäßiges Interesse an persönlichen Angelegenheiten zu Lasten von theologischen Diskussionen und innerkirchlichen Veränderungen. Kirchen teilten, was das betrifft, das Los von politischen Parteien und anderen ideologischen Institutionen, so Hooghe. Er wies auf ein Entscheidungsproblem hin, das ein Dilemma mit sich bringt: Entscheidet man sich in kirchlich-religiöser Kommunikation für häufige Auftritte von Bischöfen in Fernsehshows - oder legt man die Priorität darauf, Kommentare zu aktuellen Fragen der Gesellschaft abzugeben? Auf die erste Weise erreicht der Kirchenvertreter ein großes, aber vielleicht nur oberflächlich an religiösen Fragen interessiertes Publikum. Bei der zweiten Herangehensweise besteht die Chance, dass Personen mit einer intermediären Position in der Gesellschaft die Botschaft in ihrem eigenen Kreis aufgreifen und in angemessener Form weitergeben. Auch dieses Beispiel zeigt, dass sich über strategische Kommunikation im kirchlich-religiösen Zusammenhang noch viel diskutieren lässt.

Aus einer Untersuchung des Interesses von Journalisten an Kirche und Religion in den Niederlanden, durchgeführt von Joan Hemels (vgl. „Communicatio Socialis“40. Jg. 2007, Heft 2, S. 129-157), und in Frankreich, durchgeführt von Jacques Guyot (Universität Paris 8 - Vincennes), zogen beide Wissenschaftler die Schlussfolgerung, dass man in beiden Ländern von wachsender Aufmerksamkeit für das Religiöse sprechen kann, wenn auch vor allem losgelöst von jeglichen kirchlichen Institutionen und der Kirche als Organisation. Guyot machte anhand verschiedener Beispiele aus Sendungen des öffentlich-rechtlichen Rundfunks in Europa deutlich, dass die Trennung von Kirche und Staat kein Hindernis ist, große Aufmerksamkeit auf religiöse Themen zu richten. Jim McDonnell hingegen erläuterte, wie die britische Regierung im „Broadcasting Act" von 1990 den religiösen Rundfunkprogrammen ihren festen Sendeplatz im öffentlich-rechtlichen Rundfunksystem der British Broadcasting Corporation entzogen habe, aber der "Communications Act" 2003 neue Möglichkeiten für die Programmkategorie „Religion(en)“ bietet, unter anderem Dank der Digitalisierung.

Die Situation in Lateinamerika, Afrika und Asien thematisierte Daniela Frank vom Aachener "Catholic Media Council" in ihrem Beitrag mit Fallstudien. Sie ging von der Frage aus, was die katholische Identität des Fernsehens ausmache. Muss man, so fragte sie weiter, dabei in erster Linie Wert legen auf eine Eigentumsstruktur oder auf den Inhalt der 
Sendungen? Und wie attraktiv und relevant sind sie für die Kirche(n)? Frank fragte, wer die Zuschauer katholischer Fernsehsendungen sind und wie aus Sicht der Anbieter die Gesamtheit des christlichen Glaubens an ein vielfältiges und modern denkendes Publikums überhaupt kommuniziert werden kann.

\section{Welcher Eindruck bleibt?}

Welcher Eindruck bleibt von diesem Kongress in Antwerpen mit so vielen Rednern und genauso vielen Unterthemen? Aufschlussreich war, dass mehrere Sprecher betonten, dass die Kirchen in den meisten Ländern nicht mehr automatisch Zugang zu den Medien hätten. Sie wiesen darauf hin, dass sich die Kirchen ihre Abhängigkeit von der Gunst der Journalisten und Programmmacher eingestehen müssten, bevor sie sich auf eine aktive Kommunikationspolitik und regelmäßigen Zugang zu den Massenmedien einstellen könnten. Außerdem wurde klar, dass das Wissen über die Zielsetzung verschiedener kommerzieller und Non-Profit-Medien kirchliche Verantwortliche vor einem allzu großen Risiko schützen kann, wenn sie Kontakt zu den Medien suchen oder um Mitarbeit an Interviews oder anderen journalistischen Beiträgen gebeten werden. Auch fiel mir auf, welche Folgen die Trennungslinie zwischen Medien, die sich in "low media culture" profilieren und Medien, die sich besonders an der öffentlichen Debatte beteiligen, für die kirchliche Kommunikationspolitik hat. Diese Zweiteilung stellt Kirchenführer vor ein Wahlproblem. Sollen sie den Hang populärer Medien unterstützen, sich auf Personen und ihre Ziele, Widersprüche und Mängel zu fokussieren, oder nicht? Schließlich klang in den eher allgemein gehaltenen Referaten durch, dass die Möglichkeiten von digitalen Themenkanälen und Internetseiten entdeckt und auf die spezifischen Zielgruppen zugeschnitten werden müssen. Gleichzeitig zeigte sich dabei, dass das Bedürfnis bestehen bleibt, Kirchen und Religionen in einem Forum von Massenmedien zu präsentieren, die sich an ein gemischtes, breiteres Publikum richten.

Der Bruch zwischen der Welt der Kirche und der Medienkultur hat offenbar den Weg für eine Rückbesinnung und eine Neudefinition des Verhältnisses zueinander freigemacht. Viele Sprecher zeigten deutlich, wie sich Medien und Religionen voneinander angezogen fühlen, vor allem, wenn die kirchlichen Institutionen dabei keine überragende Rolle spielen. In einigen Vorträgen wurde der Beginn einer Konvergenz der Medienkultur und der religiösen Kultur signalisiert. Hierbei kann man insbesondere an die Aufmerksamkeit, die Zeitschriften den Themen Spiritualität und Sinngebung widmen, denken. Henau lobte Pfarrerserien 
im Fernsehen, weil darin häufig lokale Glaubensgemeinschaften positiv dargestellt würden. Historische Fernsehdokumentationen würden hingegen oft ein Bild von Beschränktheit, falschen Einschätzungen und Entscheidungen seitens der Kirchen und kirchlichen Führer vermitteln. Dadurch, dass vieles verzerrt dargestellt werde, entsteht nach Henaus Auffassung ein negatives Bild der historischen Rolle von Kirchen.

Hoover ging mit der Konvergenzthese am weitesten. Aufgrund der Suche von Individuen nach Sinn im Alltagsleben sieht er nämlich gute Chancen für ein auf diverse Zielgnuppen zugeschnittenes Angebot von verschiedenen Medien im Bereich religiöser Bedeutungsgebung. Wie Kirchen in dieser neuen Situation auf dem Markt der Sinnsuche ihr Angebot auf die Nachfrage abstimmen können, blieb ziemlich unklar. Europäer lassen sich vielleicht nicht so leicht für Fernsehgottesdienste der amerikanischen „Electronic Churches" begeistern und glauben, dass es bei Gottesdiensten nicht hauptsächlich und sicherlich nicht ausschließlich um "to comfort” gehen muss. In der europäischen Annäherung muss abgewechselt werden zwischen einer "to challenge"-Annäherung und einem „to comfort“-Anspruch. 